\title{
Evaluation of Potential Clinical Surrogate Markers of a Trauma Induced Alteration of Clotting Factor Activities
}

\author{
Manuel Burggraf, ${ }^{1}$ Arzu Payas, ${ }^{1}$ Carsten Schoeneberg, ${ }^{2}$ Alexander Wegner, \\ Max Daniel Kauther, ${ }^{1}$ and Sven Lendemans ${ }^{2}$ \\ ${ }^{1}$ Department of Orthopedics and Emergency Surgery, University Hospital Essen, University of Duisburg-Essen, \\ Hufelandstraße 55, 45147 Essen, Germany \\ ${ }^{2}$ Department of Emergency and Orthopedic Surgery, Alfried Krupp Hospital Steele, Hellweg 100, 45276 Essen, Germany
}

Correspondence should be addressed to Manuel Burggraf; manuel.burggraf@uk-essen.de

Received 15 March 2016; Accepted 6 June 2016

Academic Editor: Pedro Cabrales

Copyright ( 2016 Manuel Burggraf et al. This is an open access article distributed under the Creative Commons Attribution License, which permits unrestricted use, distribution, and reproduction in any medium, provided the original work is properly cited.

\begin{abstract}
Objective. The aim of this study was to identify routinely available clinical surrogate markers for potential clotting factor alterations following multiple trauma. Methods. In 68 patients admitted directly from the scene of the accident, all soluble clotting factors were analyzed and clinical data was collected prospectively. Ten healthy subjects served as control group. Results. Patients showed reduced activities of clotting factors II, V, VII, and X and calcium levels (all $P<0.0001$ to 0.01 ). Levels of hemoglobin and base deficit correlated moderately to highly with the activities of a number of clotting factors. Nonsurvivors and patients who needed preclinical intubation or hemostatic therapy showed significantly reduced factor activities at admission. In contrast, factor VIII activity was markedly elevated after injury in general $(P<0.0001)$, but reduced in nonsurvivors $(P<0.05)$. Conclusions. Multiple trauma causes an early reduction of the activities of nearly all soluble clotting factors in general. Initial hemoglobin and, with certain qualifications, base deficit levels demonstrated a potential value in detecting those underlying clotting factor deficiencies. Nevertheless, their role as triggers of a hemostatic therapy as well as the observed response of factor VIII to multiple trauma and also its potential prognostic value needs further evaluation.
\end{abstract}

\section{Introduction}

Multiple trauma is the most common cause of death in adolescence and young adulthood worldwide $[1,2]$. While in deleterious traumatic brain injury (TBI) treatment options are usually limited, uncontrolled hemorrhage is deemed to be the most common preventable cause of death after trauma [3]. A considerable number of studies focused on the ideal administration of combinations of packed red blood cells, fresh frozen plasma, and/or hemostatic blood products. Yet, the ideal resuscitation protocol for traumatic hemorrhage still has to be found as no single intervention besides early application of tranexamic acid showed a clear superiority [4].

Currently, only few studies exist that systematically investigated clotting factor activities early after multiple injury. Rizoli et al. reported a critical deficiency of coagulation factor activities in $20 \%$ of severely injured patients at time of admission [5]. A subgroup analysis showed that the degree of hypoperfusion as reflected by levels of base deficit is associated with reduced activity in various coagulation factors [6]. Cohen et al., based on a subgroup analysis of data from the PROMMTT-study, demonstrated that clotting factor activities are reduced in coagulopathic major trauma patients receiving blood products [7]. Furthermore, a moderate reduction of clotting factor activities early after severe multiple trauma, irrespective of patient coagulation status, was shown [8]. However, all studies excluded a considerable number of patients from the final analysis according to certain patient characteristics (e.g., injury severity). We think that those results finally have to be proven for the majority of multiple trauma patients, as deleterious bleeding disorders are obviously more likely but not limited to certain patient subgroups. Moreover, in order to achieve clinical benefits from possible findings, reliable and easily available indicators 
of clotting factor impairments have to be found. Thus, the two primary objectives of this study were to generally analyze the clotting factor activities of multiple trauma patients immediately after admission to the trauma shock room and, secondly, to identify routinely available clinical parameters which could act as surrogate markers for the underlying clotting conditions.

\section{Materials and Methods}

2.1. Patients and Normal Donors. After sustaining multiple trauma, adult patients were enrolled into the study if admitted directly from the scene of the accident to the trauma bay at a Level 1 academic trauma center in Germany. Demographic data was collected prospectively. Ten healthy adult volunteers served as the control group. Patients with preexisting congenital or acquired coagulopathies and pregnant women were excluded from the study. The study was carried out in compliance with the Helsinki Declaration and was approved by the relevant local ethics committee (reference 12-5120-BO). Written informed consent was obtained from the participants. This study expands data from an original prospective study of severely injured patients by minor to moderate multiple trauma victims [8].

2.2. Blood Samples. Blood samples were drawn from a femoral artery directly after admission to the resuscitation room. Blood from healthy controls was drawn from a cubital vein. Blood samples were immediately transferred to the hospital laboratory. Besides standard blood tests, the activities of all remaining soluble clotting factors (FII, FV, FVII, FVIII, FIX, FX, FXI, FXII, and FXIII) were analyzed. The results are expressed as a percentage of standard activity. If the testing of clotting factor activity was not available right away (mainly due to off-duty hours), the specimens were cryostored at $-70^{\circ} \mathrm{C}$ and analyzed the following weekday.

2.3. Statistical Analysis. IBM ${ }^{\circledR}$ SPSS ${ }^{\circledR}$ Statistics (Version 20) was used for statistical analysis and to compile the graphs. The $t$-test (age) and Fisher's exact test (gender) were used to determine demographic differences between patients and healthy volunteers. Differences between clotting factor activities of patients and controls were analyzed by using the MannWhitney $U$-test. Secondly, potential relationships between the clotting factor activities of traumatized patients and the patients' demographic and clinical data were investigated. For all scale and most ordinate variables Spearman's rank correlation, coefficient rho $(\rho)$ was calculated to reveal possible relationships. Additionally, the $95 \%$ confidence interval (CI) was computed by bootstrapping using a bias-corrected and accelerated method based on 1000 bootstrap samples. The correlation was considered negligible for absolute values of $\rho$ between 0.0 and 0.2 , weak between 0.21 and 0.4 , moderate between 0.41 and 0.7 , strong between 0.71 and 0.9 , and very strong between 0.91 and 1 . When analyzing differences between distinct patient subgroups, the Mann-Whitney $U$ test was used. The subgroups were defined by gender, survival, preclinical fluid volume ( $<$ or $\geq 1500 \mathrm{~mL}$ ), preclinical intubation, and administration of hemostatic agents
TABLE 1: Demographic data of patients. Data reported as mean \pm SD or absolute value and percentage of the study group. Data acquisition upon arrival if not otherwise specified. Volume replacement refers to crystalloids and/or colloids. ISS: injury severity score; TBI: traumatic brain injury.

\begin{tabular}{|c|c|c|}
\hline \multicolumn{3}{|c|}{ Patients $(n=68)$} \\
\hline Age & $45 \pm 17$ & Years \\
\hline Gender & $56(82 \%)$ & Male \\
\hline ISS & $24 \pm 13$ & Points \\
\hline \multirow{3}{*}{ Mechanism of trauma } & $59(87 \%)$ & Blunt \\
\hline & $3(4 \%)$ & Penetrating \\
\hline & $6(9 \%)$ & Isolated TBI \\
\hline Mortality & $13(19 \%)$ & \\
\hline Systolic blood pressure (preclinical) & $131 \pm 34$ & $\mathrm{mmHg}$ \\
\hline Systolic blood pressure (admission) & $132 \pm 26$ & $\mathrm{mmHg}$ \\
\hline Lactate & $2.3 \pm 1.8$ & $\mathrm{mmol} / \mathrm{L}$ \\
\hline Base deficit & $-2.8 \pm 3.8$ & $\mathrm{mmol} / \mathrm{L}$ \\
\hline Hemoglobin & $12.4 \pm 2.2$ & $\mathrm{mg} / \mathrm{dL}$ \\
\hline Temperature & $35.8 \pm 0.9$ & ${ }^{\circ} \mathrm{C}$ \\
\hline Volume replacement (preclinical) & $1048 \pm 563$ & $\mathrm{~mL}$ \\
\hline Intubation (preclinical) & $29(43 \%)$ & \\
\hline Time to admission & $63 \pm 23$ & Minutes \\
\hline
\end{tabular}

(fibrinogen, tranexamic acid, prothrombin complex, and/or factor concentrates) within the hospital stay. Accordingly, a Kruskal-Wallis (one-way ANOVA by ranks) test was used to detect differences between three distinct trauma entities (blunt, penetrating, and isolated TBI). A $P$ value smaller than 0.05 (2-tailed) was considered statistically significant for all tests. Demographic data is reported as means and standard deviation (SD), whereas results are reported as median values. All authors had access to primary clinical data.

\section{Results}

3.1. Demographic Data. 68 multiple injured patients were enrolled into the final study. The mean age of $45 \pm 17$ years did not significantly differ from the control group (40 \pm 9 years, $P=0.15$ ). Overall, $82 \%$ of patients and $70 \%$ of healthy controls were male. Statistical analysis showed no significant difference in gender between both groups $(P=0.40)$. Patients showed a mean ISS of $24 \pm 13$ points and were admitted to the resuscitation room $63 \pm 23$ minutes after trauma. 59 patients suffered from blunt trauma, six from isolated TBI, and three from penetrating trauma. 13 patients showed a fatal outcome. Full demographic data is presented in Table 1.

3.2. Standard Coagulation Tests. Routinely performed coagulation tests showed significant changes following multiple trauma (Figures 1-4). The patients' International Normalized Ratio (INR) was elevated (1.06 versus $0.96, P=0.001$ ) whereas the Partial Thromboplastin Time (PTT) was shortened (25.3 versus 28.8 seconds, $P<0.05$ ) compared to controls. Serum levels of calcium were reduced as well $(2.13$ versus $2.30 \mathrm{mmol} / \mathrm{L}, P<0.0001)$. Fibrinogen showed a trend towards decreased levels. However, this reduction did 


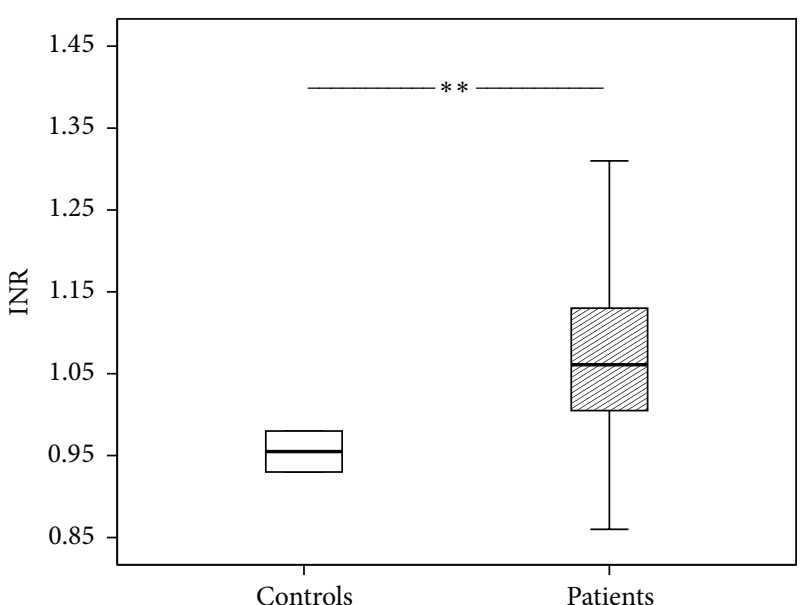

FIGURE 1: International Normalized Ratio (INR) of patients and control group. The results are presented by using boxplots. Bottom and top of the box indicate the 25th and 75th percentile, called interquartile range (IR). The median is represented by the horizontal bar within the box. Whiskers indicate spread (1.5 times IR). The Mann-Whitney $U$-test was performed with ${ }^{* *} P=0.001$.

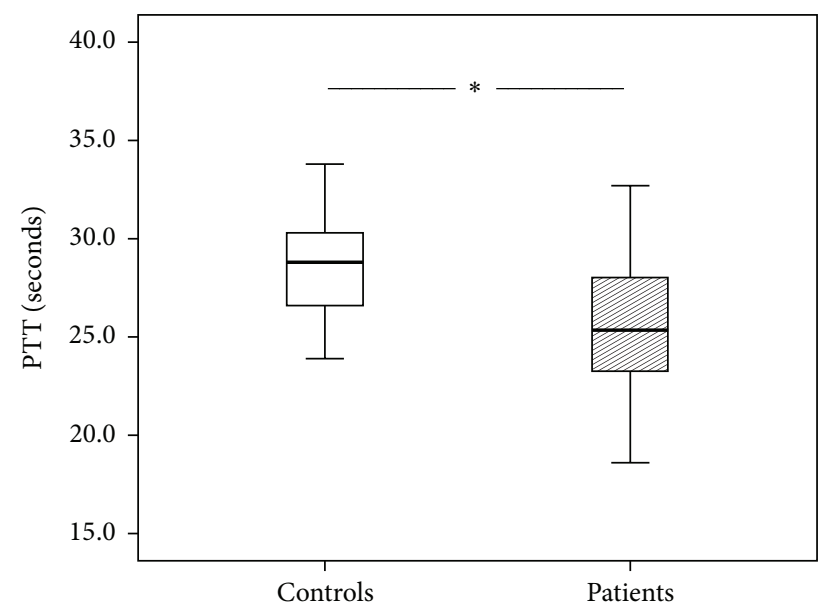

FIgUre 2: Partial Thromboplastin Time (PTT) of patients and control group. The results are presented by using boxplots. Bottom and top of the box indicate the 25th and 75th percentile, called interquartile range (IR). The median is represented by the horizontal bar within the box. Whiskers indicate spread (1.5 times IR). The Mann-Whitney $U$-test was performed with ${ }^{*} P<0.05$.

not reach statistical significance $(237$ versus $296 \mathrm{mg} / \mathrm{dL}, P=$ $0.08)$.

3.3. Coagulation Factor Activity. Figure 5 shows the activities of the remaining soluble clotting factors. Final analysis showed significantly reduced activities of FII ( 82 versus $122 \%$, $P<0.0001)$, FV (80 versus 123\%, $P<0.0001)$, FVII $(90$ versus $114 \%, P<0.01$ ), and FX (88 versus $122 \%, P<0.0001)$ whereas the activity of FVIII was elevated significantly (208 versus $109 \%, P<0.0001$ ). The remaining factors showed a trend towards reduced activity and serum levels, respectively.

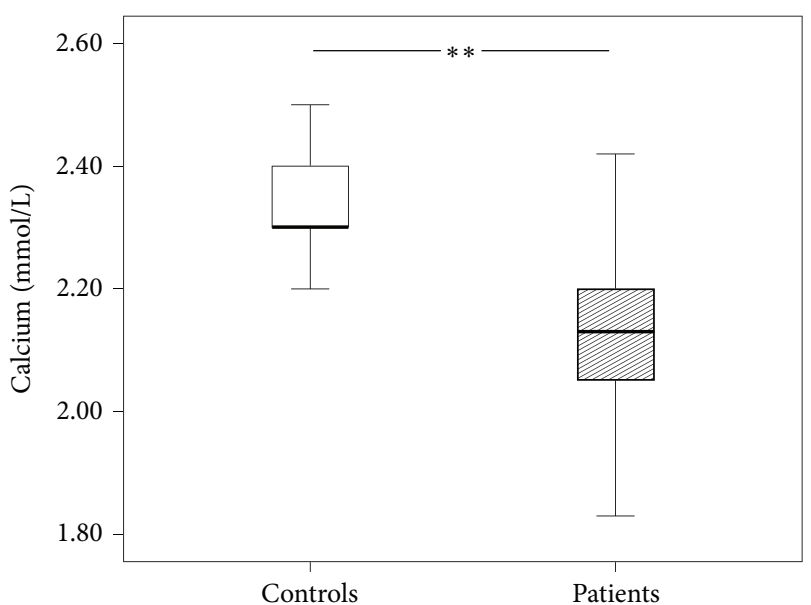

FIGURE 3: Calcium levels of patients and control group. The results are presented by using boxplots. Bottom and top of the box indicate the 25 th and 75 th percentile, called interquartile range (IR). The median is represented by the horizontal bar within the box. Whiskers indicate spread (1.5 times IR). The Mann-Whitney $U$-test was performed with ${ }^{* *} P<0.0001$.

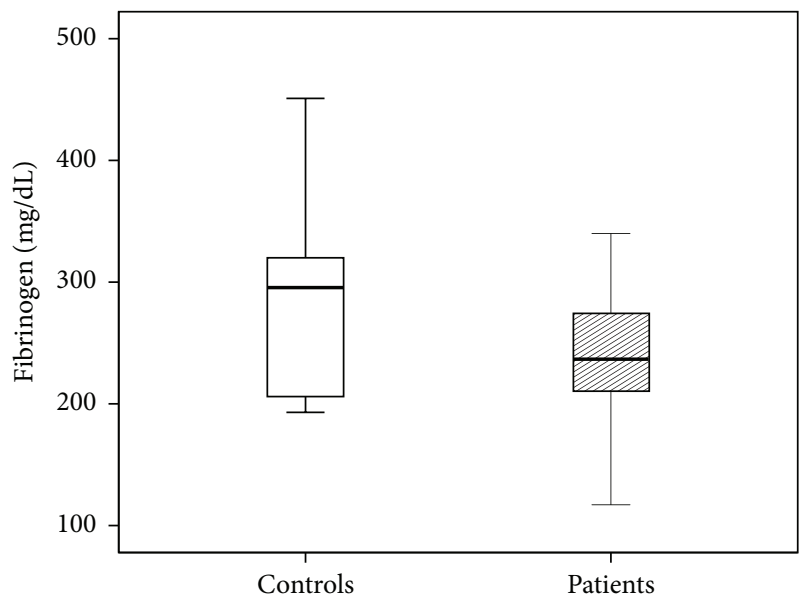

Figure 4: Fibrinogen levels of patients and control group. The results are presented by using boxplots. Bottom and top of the box indicate the 25 th and 75 th percentile, called interquartile range (IR). The median is represented by the horizontal bar within the box. Whiskers indicate spread (1.5 times IR). The Mann-Whitney $U$-test was performed to test for differences.

3.4. Correlation Analysis. Rho and 95\% confidence intervals for the relationship between clotting factors and the results of routine blood tests are given in Table 2. INR showed a moderate to strong negative correlation with fibrinogen levels and the activities of FII, FV, FVII, FIX, and FX. Furthermore, PTT showed a moderate to strong negative correlation with the activities of FII, FV, FVIII, FIX, FXI, and FXII. Levels of hemoglobin were also moderately related to a series of clotting factors (FII, FV, FIX, FX, FXI, FXII, and calcium). In addition, platelet counts (for FII and FXI) as well as BD (for FII, FV, and calcium) showed a few moderate correlations among the pairings. In contrast, levels 


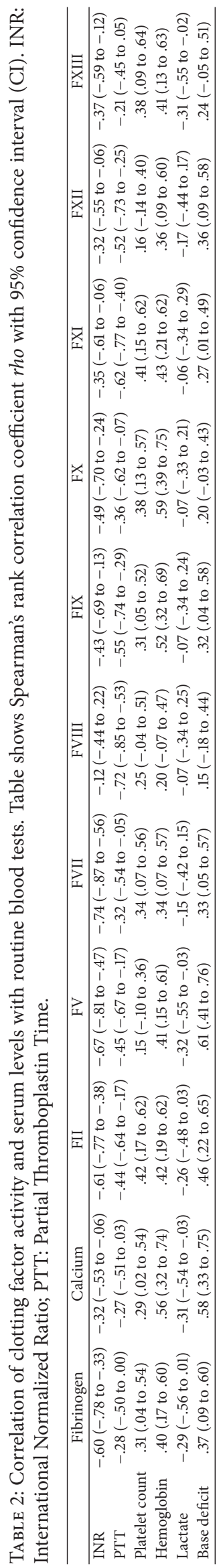




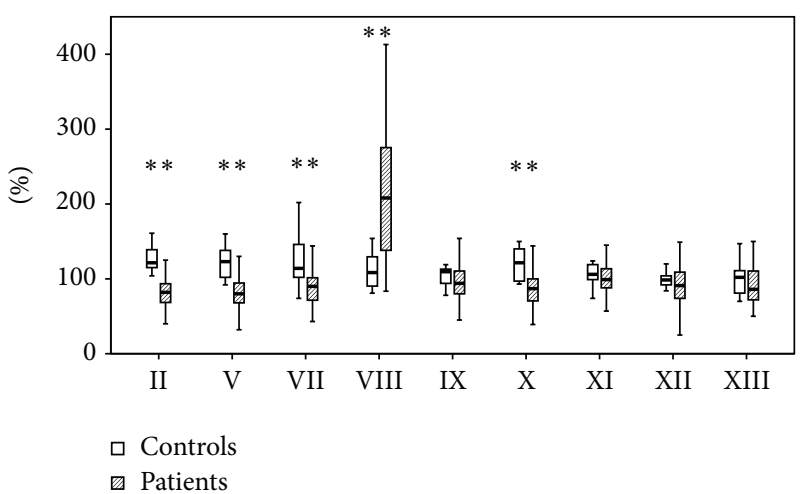

FIGURE 5: Clotting factor activities of patients and control group. The results are presented by using boxplots. Bottom and top of the box indicate the 25th and 75th percentile, called interquartile range (IR). The median is represented by the horizontal bar within the box. Whiskers indicate spread (1.5 times IR). The Mann-Whitney $U$-test was performed with ${ }^{* *} P<0.01$.

of lactate did not show any relevant correlation at all. Table 3 presents the relationships between clotting factors and selected demographic data. Final analysis showed moderate negative correlations between the injury severity score (ISS) and FIX and FXI. Moreover, the preclinical systolic blood pressure (SBP) correlated with the activities of FV, FIX, and FXII and the SBP at admission was related to FII, FIX, and FXI. Additionally, the time until admission to hospital showed a negative relationship with the activities of FIX and FXI. Finally, no relevant correlations between the levels and activities of clotting factors and the body temperature at admission or the age of the patients were found. All moderate to high correlations reached statistical significance $(P<$ 0.0001 to 0.01 ).

3.5. Subgroup Analysis. Gender specific analysis did not show any significant differences in clotting factor activity between male and female patients. Analysis of the varying trauma entities blunt, isolated TBI, and penetrating showed significant differences among the three groups only for FXIII activity, which was significantly reduced after penetrating trauma ( 88 versus 69 versus 51\%, $P<0.01$ ).

Figures 6-9 present the differences related to survival, intubation, hemostatic therapy, and prehospital volume replacement. In contrast to survivors, nonsurvivors had significantly reduced activities of FII (66 versus $84 \%, P<$ 0.01 ), $\mathrm{FV}$ (54 versus $84 \%, P<0.01$ ), FVIII (141 versus $231 \%$, $P<0.05$ ), and FXIII (69 versus $88 \%, P<0.05$ ). If patients needed intubation prior to admission, the serum levels of fibrinogen (222 versus $242 \mathrm{mg} / \mathrm{dL}, P<0.01$ ) and calcium (2.06 versus $2.20 \mathrm{mmol} / \mathrm{L}, P<0.01)$ as well as the activities of FII (75 versus 90\%, $P<0.01$ ), FV (71 versus $88 \%, P<$ 0.01 ), FVIII (164 versus $237 \%, P<0.05$ ), FIX (83 versus $103 \%, P<0.01$ ), FX (85 versus $92 \%, P<0.01$ ), FXI (91 versus $108 \%, P<0.01$ ), and FXII (79 versus $99 \%, P<0.01$ ) were reduced significantly. Patients who were in need for hemostatic therapy over the course had significantly reduced levels and activities of all soluble clotting factors but FVII (71

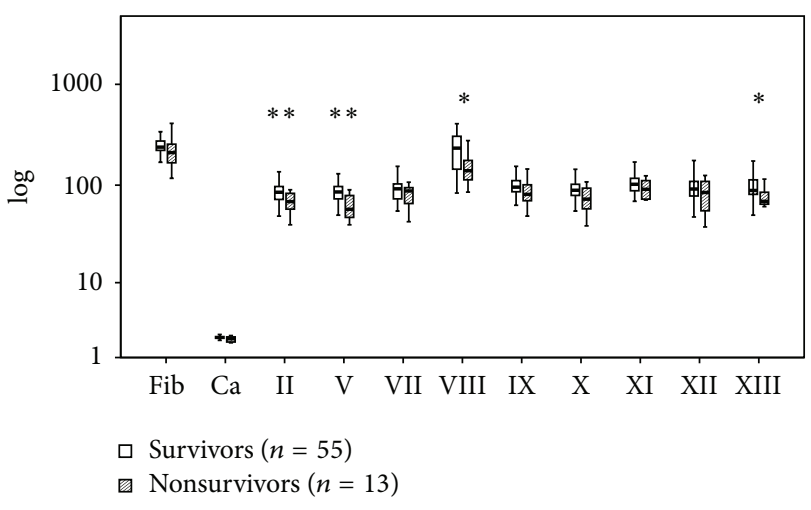

FIGURE 6: Clotting factor activities of survivors and nonsurvivors after multiple trauma. Fib: fibrinogen; Ca: calcium. The results are presented by using boxplots. Bottom and top of the box indicate the 25 th and 75 th percentile, called interquartile range (IR). The median is represented by the horizontal bar within the box. Whiskers indicate spread (1.5 times IR). Logarithmic ordinate for better depiction. The Mann-Whitney $U$-test was performed with ${ }^{*} P<0.05$ and ${ }^{* *} P<0.01$ (survivors versus nonsurvivors).

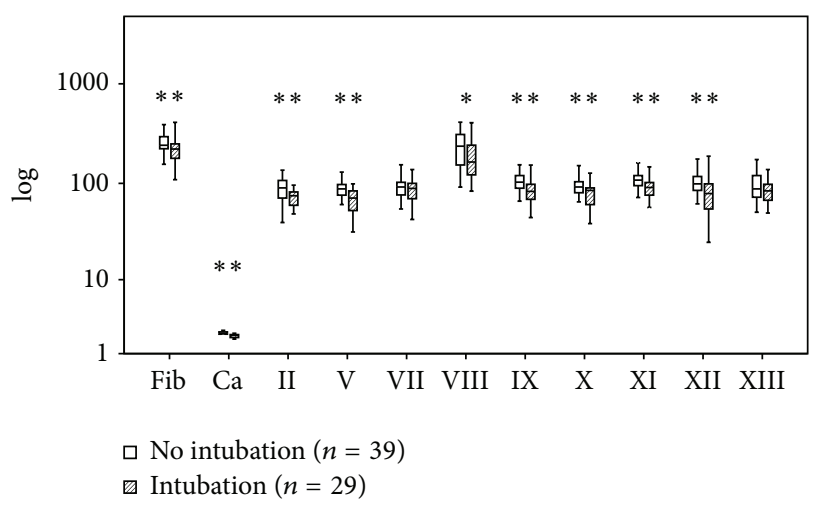

FIgURE 7: Clotting factor activities after multiple trauma and preclinical intubation. Fib: fibrinogen; $\mathrm{Ca}$ : calcium. The results are presented by using boxplots. Bottom and top of the box indicate the 25 th and 75 th percentile, called interquartile range (IR). The median is represented by the horizontal bar within the box. Whiskers indicate spread (1.5 times IR). Logarithmic ordinate for better depiction. The Mann-Whitney $U$-test was performed with ${ }^{*} P<0.05$ and ${ }^{* *} P<0.01$ (intubation versus nonintubation).

versus $91 \%, P=0.06$ ), FVIII (151 versus $216 \%, P=0.35$ ), and FXIII (73 versus $93 \%, P=0.05$ ). If the amount of prehospital volume replacement exceeded $1500 \mathrm{~mL}$, levels of fibrinogen (203 versus $237 \mathrm{mg} / \mathrm{dL}, P<0.01)$ and calcium $(2.06$ versus $2.16 \mathrm{mmol} / \mathrm{L}, P<0.01)$ as well as activities of FII (77 versus $87 \%, P<0.05$ ), FVII ( 66 versus $92 \%, P<0.05$ ), $\mathrm{FX}$ ( 77 versus $90 \%, P<0.01$ ), FXI (91 versus 108\%, $P<0.05$ ), and FXII ( 87 versus $99 \%, P<0.05)$ were decreased significantly.

\section{Discussion}

After multiple trauma, the activities of FII, FV, FVII, FX, and calcium levels are significantly reduced. In principle, this data should reflect the majority of multiple trauma patients, 


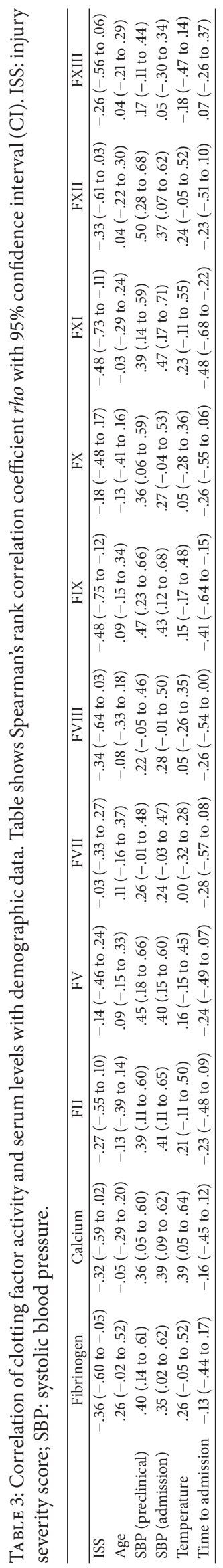




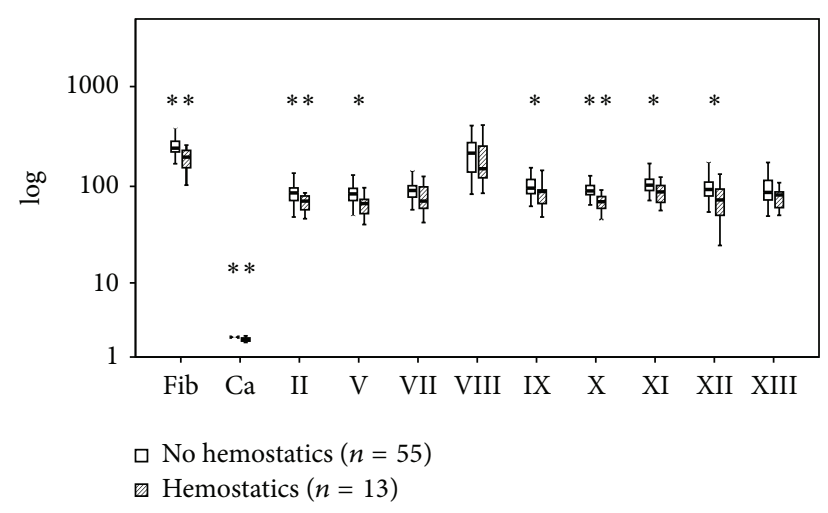

FIGURE 8: Clotting factor activities at admission after multiple trauma and the need for hemostatic therapy. Fib: fibrinogen; Ca: calcium. The results are presented by using boxplots. Bottom and top of the box indicate the 25 th and 75th percentile, called interquartile range (IR). The median is represented by the horizontal bar within the box. Whiskers indicate spread (1.5 times IR). Logarithmic ordinate for better depiction. The Mann-Whitney $U$-test was performed with ${ }^{*} P<0.05$ and ${ }^{* *} P<0.01$ (hemostatic versus nonhemostatic therapy within hospital stay).

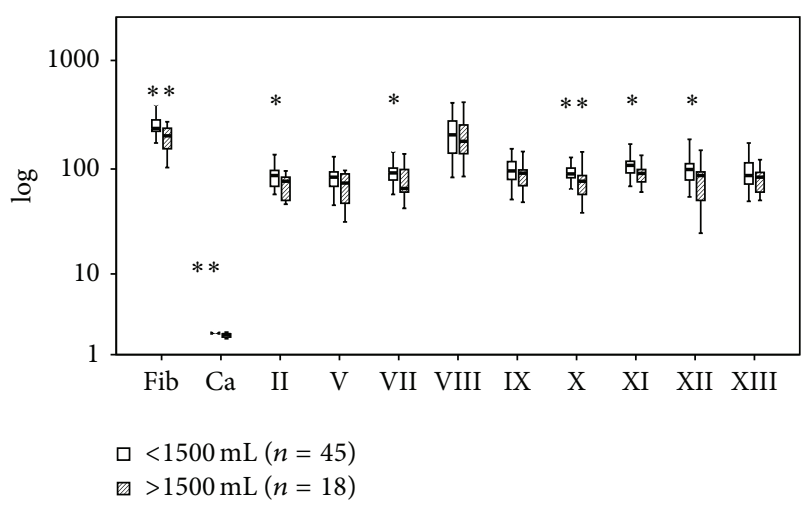

FIGURE 9: Clotting factor activities after multiple trauma according to amount of preclinical fluid resuscitation. Fib: fibrinogen; Ca: calcium. The results are presented by using boxplots. Bottom and top of the box indicate the 25 th and 75 th percentile, called interquartile range (IR). The median is represented by the horizontal bar within the box. Whiskers indicate spread (1.5 times IR). Logarithmic ordinate for better depiction. The Mann-Whitney $U$-test was performed with ${ }^{*} P<0.05$ and ${ }^{* *} P<0.01(<1500$ versus $>1500 \mathrm{~mL}$ of preclinical fluid volume).

as no exclusion criteria in terms of preexisting treatment, ISS, or the subsequent clinical course were used. It must be said, though, that the reduction of clotting factor activities, although statistically significant, is rather small. None of the median activities falls short of the lower limits of its associated reference range ( $70 \%$, as given by the laboratory). The results basically reflect findings in severely injured patients, but the measured activities are clearly less impaired if compared to highly selected patient cohorts (e.g., coagulopathic and/or in shock) [5-8]. Nevertheless, we think that the observed clotting factor activities raise the question whether simple administration of fresh frozen plasma is the best treatment option when attempting to correct suspected clotting factor deficiencies. There is growing experimental and clinical evidence that resuscitation fluids with higher ratios of clotting factors such as prothrombin complex concentrate might be beneficial in this context [9-11].

When looking for markers of impaired factor activities, the initial hemoglobin level appears to be a valuable indicator. This is in conjunction with the recent finding that hemoglobin acts very well as a trigger for initiating a coagulation therapy $[12,13]$. As shock is considered to be the prime driver of a trauma induced coagulopathy (TIC) [14], we also assessed the prognostic value of different shock markers (SBP, BD, and lactate). The observed discrepancy in the disadvantage of lactate might partially explain why BD was a better predictor of traumatic coagulopathy in a retrospective analysis [15]. Hence, also various relationships with factor activities were found for $\mathrm{BD}$ (as for pre- and intrahospital SBP); strength of these relationships was generally of lesser extent and appeared rather randomly distributed. Nevertheless, the moderate correlation of BD with FII activity might be one explanation for the described relationship of initial base deficit values with subsequent transfusion requirements and also mortality in severely injured patients [16]. Thrombin, the proteolytically activated form of FII, is a crucial protease in the process of coagulation [17], and, indeed, in our study the activity of FII was significantly reduced in nonsurvivors. Excessive preclinical fluid resuscitation $(>1500 \mathrm{~mL})$ led to significantly reduced clotting factor activities and, not surprisingly, patients with reduced clotting factor activities needed hemostatic agents significantly more often. Although dilution is not any longer seen as the primary contributor to TIC, this underlines the need for a rational application of fluids not to aggravate coagulopathy and outcome as shown by Hussmann et al. [18, 19]. Finally, we found broadly reduced clotting factor activities after preclinical intubation. Need for intubation at the scene of an accident usually indicates higher injury severity and might lead to a prolonged time to admission. Hence, the origin of this relationship ultimately remains unclear, as neither ISS nor time to admission and also not body temperature demonstrated such a clear effect on the activity of soluble clotting factors, as it could be expected from the literature $[15,20]$.

Moreover, analysis showed a fair relationship of both INR and PTT, with a series of clotting factor activities early after multiple trauma. Yet, we cannot recommend those tests in general as both have well-known limitations [21]. In our study, multiple trauma led to a significant rise of the INR, reflecting a mild coagulopathic state of the patient cohort as a whole, whereas, in contrast, the observed PTT was significantly shortened. This fact might explain why some studies report higher rates of coagulopathic patients when taking INR (or Prothrombin Time) instead of PTT as criterion for coagulopathy $[7,22,23]$. We have previously hypothesized that the observed high levels of FVIII after severe trauma might influence the in vitro assay method for PTT measurement in terms of a reduced interval until clot formation [8]. Indeed, it was shown that high levels of FVIII, for example, in so-called heparin resistance, can lead to a shortened PTT [24-26]. This effect seems to be irrespective 
of the commercial reagent used for testing [27]. Importantly, the activity of FVIII in trauma patients seems to react rather contrary to the activities of the remaining factors [6-8]. Therefore, diagnosis of an overall coagulopathic state might be missed when focusing on PTT values in trauma patients. Interestingly, FVIII concentrations also strongly correlate with results of point-of-care viscoelastic tests [28]. Evidence from in vitro experimental heparin resistance due to high levels of FVIII indicates that elevation of FVIII activity could eventually bias viscoelastic tests as well [29]. Unfortunately, as use of viscoelastic tests is not yet routinely implemented in the trauma bay algorithm at our institution, we lack of sufficient data to evaluate this potentially relevant bias in a clinical setting.

The likeliest explanation of the posttraumatic elevation of FVIII activity is an acute phase reaction, although further mechanisms like liberation from injured tissues are conceivable, as FVIII is produced by a variety of cell types [30-32]. However, the (patho)physiological meaning of the elevated FVIII activity is currently unknown. When circulating in plasma, FVIII is bound to von Willebrand factor as a carrier protein. It is involved in the amplification and propagation of the coagulation process [17]. This effect is mediated via complex interactions, mainly based on specific binding and consecutive activation of platelets [17, 33-35]. A relative deficiency of FVIII, for example, as seen in nonsurvivors, might explain a recently described posttraumatic platelet dysfunction [36-39]. If true, this would indicate a true physiological role of the elevated FVIII activity. On the other hand, a posttraumatic FVIII elevation might also possess negative implications. So far, several studies showed that high FVIII levels are an independent risk factor for venous thrombosis [40]. Among other numerous risk factors, this could at least partially explain the high susceptibility of multiple trauma patients for a variety of thromboembolic complications [41].

This study comprises some important limitations. They include study design, specimen handling, confounding due to individualized pre- and intrahospital patient care, and generalizability in principle. Results of this study are based on a rather small study size from a single trauma center. Therefore, findings have to be questioned critically as larger study populations might have unveiled different results. This is especially true for the different trauma entities, as the incidence of blunt traumata outweighs penetrating injuries by far. Supposing that penetrating injuries may rather lead to hemorrhage compared to vast tissue traumatization, this difference might have a divergent impact on coagulation in general and clotting factor activity in particular. This potential influence of patient demographics was discussed previously, as it represents the medical reality in Germany but may lead to divergent results in populations with significantly higher rates of penetrating trauma $[8,42]$. On the other hand, except for the studies of Cohen, Rizoli, and Jansen investigating 165, 110, and 71 selective patients, investigations based on large patient cohorts are still missing [5-7]. Specimen handling itself bears a risk due to different half-lives until analysis, but this is highly unlikely and the procedure is widely accepted [8]. Another important limitation of this study is the strict focus on the role of soluble clotting factors. However, hemostasis is not solely based on coagulation factors, but a rather dynamic process involving various cells until final clot formation [17]. Hence, we think that profound knowledge of clotting factor derangements early after trauma is inevitable to conceptualize well-founded strategies for hemostatic resuscitation.

\section{Conclusions}

Multiple trauma predominantly leads to an early reduction of activities of soluble clotting factors. However, this loss of activity is of rather small extent overall. In contrast, FVIII levels are markedly elevated after injury and this may influence standard PTT measurements. Initial hemoglobin and, with certain qualifications, base deficit levels demonstrated a potential value in detecting underlying clotting factor deficiencies, whereas neither ISS, body temperature, nor time to admission did show a clear relationship. Nevertheless, implementation of results into clinical pathways needs substantially larger study sizes and a multicenter approach.

\section{Competing Interests}

The authors declare that they have no competing interests.

\section{Authors' Contributions}

Manuel Burggraf conducted the study and drafted the paper. Sven Lendemans designed and supervised the study. Arzu Payas included patients and helped in analyzing and interpreting the data. Carsten Schoeneberg, Alexander Wegner, and Max Daniel Kauther analyzed the results, made the figures, and critically reviewed the paper. All authors had access to primary clinical data and read and approved the final paper.

\section{Acknowledgments}

The study was financed by the Department of Orthopedics and Emergency Surgery, University Hospital Essen, University of Duisburg-Essen, Germany.

\section{References}

[1] H. K. Herbert, A. A. Hyder, A. Butchart, and R. Norton, "Global health: injuries and violence," Infectious Disease Clinics of North America, vol. 25, no. 3, pp. 653-668, 2011.

[2] G. C. Patton, C. Coffey, S. M. Sawyer et al., "Global patterns of mortality in young people: a systematic analysis of population health data," The Lancet, vol. 374, no. 9693, pp. 881-892, 2009.

[3] R. L. Gruen, G. J. Jurkovich, L. K. McIntyre, H. M. Foy, and R. V. Maier, "Patterns of errors contributing to trauma mortality: lessons learned from 2594 deaths," Annals of Surgery, vol. 244, no. 3, pp. 371-380, 2006.

[4] N. Curry, S. Hopewell, C. Dorée, C. Hyde, K. Brohi, and S. Stanworth, "The acute management of trauma hemorrhage: a systematic review of randomized controlled trials," Critical Care, vol. 15, no. 2, article R92, 2011. 
[5] S. B. Rizoli, S. Scarpelini, J. Callum et al., "Clotting factor deficiency in early trauma-associated coagulopathy," Journal of Trauma-Injury, Infection and Critical Care, vol. 71, no. 5, supplement 1, pp. S427-S434, 2011.

[6] J. O. Jansen, S. Scarpelini, R. Pinto, H. C. Tien, J. Callum, and S. B. Rizoli, "Hypoperfusion in severely injured trauma patients is associated with reduced coagulation factor activity," Journal of Trauma-Injury, Infection and Critical Care, vol. 71, no. 5, supplement 1, pp. S435-S440, 2011.

[7] M. J. Cohen, M. Kutcher, B. Redick et al., "Clinical and mechanistic drivers of acute traumatic coagulopathy," Journal of Trauma and Acute Care Surgery, vol. 75, no. 1, supplement 1, pp. S40-S47, 2013.

[8] M. Burggraf, A. Payas, M. D. Kauther, C. Schoeneberg, and S. Lendemans, "Evaluation of clotting factor activities early after severe multiple trauma and their correlation with coagulation tests and clinical data," World Journal of Emergency Surgery, vol. 10, article 435, 2015.

[9] G. Dickneite and I. Pragst, "Prothrombin complex concentrate vs fresh frozen plasma for reversal of dilutional coagulopathy in a porcine trauma model," British Journal of Anaesthesia, vol. 102, no. 3, pp. 345-354, 2009.

[10] H. Schöchl, U. Nienaber, M. Maegele et al., “Transfusion in trauma: thromboelastometry-guided coagulation factor concentrate-based therapy versus standard fresh frozen plasmabased therapy," Critical Care, vol. 15, no. 2, p. R83, 2011.

[11] P. Innerhofer, I. Westermann, H. Tauber et al., "The exclusive use of coagulation factor concentrates enables reversal of coagulopathy and decreases transfusion rates in patients with major blunt trauma," Injury, vol. 44, no. 2, pp. 209-216, 2013.

[12] P. Hilbert, G. O. Hofmann, R. Lefering, and M. F. Struck, "Trauma bay haemoglobin level: predictor of coagulation disorder in major trauma," Der Unfallchirurg, vol. 118, no. 7, pp. 601-606, 2015.

[13] P. Hilbert, G. O. Hofmann, J. Teichmann, M. F. Struck, and R. Stuttmann, "The 'coagulation box' and a new hemoglobindriven algorithm for bleeding control in patients with severe multiple traumas," Archives of Trauma Research, vol. 2, no. 1, pp. 3-10, 2013.

[14] K. Brohi, M. J. Cohen, M. T. Ganter et al., "Acute coagulopathy of trauma: hypoperfusion induces systemic anticoagulation and hyperfibrinolysis," Journal of Trauma-Injury, Infection and Critical Care, vol. 64, no. 5, pp. 1211-1217, 2008.

[15] S. Cheddie, D. J. J. Muckart, and T. C. Hardcastle, "Base deficit as an early marker of coagulopathy in trauma," South African Journal of Surgery, vol. 51, no. 3, pp. 88-90, 2013.

[16] M. Mutschler, U. Nienaber, T. Brockamp et al., "Renaissance of base deficit for the initial assessment of trauma patients: a base deficit-based classification for hypovolemic shock developed on data from 16,305 patients derived from the TraumaRegister DGU ${ }^{5}$," Critical Care, vol. 17, no. 2, article R42, 2013.

[17] M. Hoffman and D. M. Monroe III, "A cell-based model of hemostasis," Thrombosis and Haemostasis, vol. 85, no. 6, pp. 958-965, 2001.

[18] B. Hussmann, R. Lefering, C. Waydhas et al., "Does increased prehospital replacement volume lead to a poor clinical course and an increased mortality? A matched-pair analysis of 1896 patients of the Trauma Registry of the German Society for Trauma Surgery who were managed by an emergency doctor at the accident site," Injury, vol. 44, no. 5, pp. 611-617, 2013.

[19] B. Hussmann, R. Lefering, M. D. Kauther, S. Ruchholtz, P. Moldzio, and S. Lendemans, "Influence of prehospital volume replacement on outcome in polytraumatized children," Critical Care, vol. 16, no. 5, article R201, 2012.

[20] H. Lier, B. W. Böttiger, J. Hinkelbein, H. Krep, and M. Bernhard, "Coagulation management in multiple trauma: a systematic review," Intensive Care Medicine, vol. 37, no. 4, pp. 572-582, 2011.

[21] D. Fries, P. Innerhofer, and W. Schobersberger, "Time for changing coagulation management in trauma-related massive bleeding," Current Opinion in Anaesthesiology, vol. 22, no. 2, pp. 267-274, 2009.

[22] J. B. A. MacLeod, M. Lynn, M. G. McKenney, S. M. Cohn, and M. Murtha, "Early coagulopathy predicts mortality in trauma," The Journal of Trauma, vol. 55, no. 1, pp. 39-44, 2003.

[23] K. Brohi, J. Singh, M. Heron, and T. Coats, "Acute traumatic coagulopathy," Journal of Trauma-Injury, Infection and Critical Care, vol. 54, no. 6, pp. 1127-1130, 2003.

[24] M. N. Levine, J. Hirsh, M. Gent et al., "A randomized trial comparing activated thromboplastin time with heparin assay in patients with acute venous thromboembolism requiring large daily doses of heparin," Archives of Internal Medicine, vol. 154, no. 1, pp. 49-56, 1994.

[25] E. Ten Boekel and P. Bartels, "Abnormally short activated partial thromboplastin times are related to elevated plasma levels of TAT, F1+2, D-dimer and FVIII:C", Pathophysiology of Haemostasis and Thrombosis, vol. 32, no. 3, pp. 137-142, 2002.

[26] W. Z. Abdullah, S. K. Moufak, Z. Yusof, M. S. Mohamad, and I. M. Kamarul, "Shortened activated partial thromboplastin time, a hemostatic marker for hypercoagulable state during acute coronary event," Translational Research, vol. 155, no. 6, pp. 315319, 2010.

[27] E. Ten Boekel, M. Böck, G.-J. Vrielink, R. Liem, H. Hendriks, and W. de Kieviet, "Detection of shortened activated partial thromboplastin times: an evaluation of different commercial reagents," Thrombosis Research, vol. 121, no. 3, pp. 361-367, 2007.

[28] O. M. Theusinger, C. M. Schröder, J. Eismon et al., “The influence of laboratory coagulation tests and clotting factor levels on rotation thromboelastometry $\left(\right.$ ROTEM $\left.^{\circledR}\right)$ during major surgery with hemorrhage," Anesthesia and Analgesia, vol. 117, no. 2, pp. 314-321, 2013.

[29] J. Uprichard, R. A. Manning, and M. A. Laffan, "Monitoring heparin anticoagulation in the acute phase response," British Journal of Haematology, vol. 149, no. 4, pp. 613-619, 2010.

[30] M. Begbie, C. Notley, S. Tinlin, L. Sawyer, and D. Lillicrap, “The factor VIII acute phase response requires the participation of $\mathrm{NF} \kappa \mathrm{B}$ and C/EBP," Thrombosis and Haemostasis, vol. 84, no. 2, pp. 216-222, 2000.

[31] M. J. Hollestelle, T. Thinnes, K. Crain et al., "Tissue distribution of factor VIII gene expression in vivo-a closer look," Thrombosis and Haemostasis, vol. 86, no. 3, pp. 855-861, 2001.

[32] M. Jacquemin, A. Neyrinck, M. I. Hermanns et al., "FVIII production by human lung microvascular endothelial cells," Blood, vol. 108, no. 2, pp. 515-517, 2006.

[33] M. E. Nesheim, E. Furmaniak-Kazmierczak, C. Henin, and G. Cote, "On the existence of platelet receptors for factor V(a) and factor VIII(a)," Thrombosis and Haemostasis, vol. 70, no. 1, pp. 80-86, 1993.

[34] S. S. Ahmad, J. M. Scandura, and P. N. Walsh, "Structural and functional characterization of platelet receptor-mediated factor VIII binding," The Journal of Biological Chemistry, vol. 275, no. 17, pp. 13071-13081, 2000.

[35] A. Obergfell, A. Sturm, C. P. Speer, U. Walter, and R. Grossmann, "Factor VIII is a positive regulator of platelet function," Platelets, vol. 17, no. 7, pp. 448-453, 2006. 
[36] C. Solomon, S. Traintinger, B. Ziegler et al., "Platelet function following trauma; A multiple electrode aggregometry study," Thrombosis and Haemostasis, vol. 106, no. 2, pp. 322-330, 2011.

[37] M. V. Wohlauer, E. E. Moore, S. Thomas et al., "Early platelet dysfunction: an unrecognized role in the acute coagulopathy of trauma," Journal of the American College of Surgeons, vol. 214, no. 5, pp. 739-746, 2012.

[38] M. E. Kutcher, B. J. Redick, R. C. McCreery et al., "Characterization of platelet dysfunction after trauma," Journal of Trauma and Acute Care Surgery, vol. 73, no. 1, pp. 13-19, 2012.

[39] N. A. Windeløv, A. M. Sørensen, A. Perner et al., "Platelet aggregation following trauma: a prospective study," Blood Coagulation and Fibrinolysis, vol. 25, no. 1, pp. 67-73, 2014.

[40] I. Martinelli, "Von Willebrand factor and factor VIII as risk factors for arterial and venous thrombosis," Seminars in Hematology, vol. 42, no. 1, pp. 49-55, 2005.

[41] D. F. McLaughlin, C. E. Wade, H. R. Champion, J. Salinas, and J. B. Holcomb, "Thromboembolic complications following trauma," Transfusion, vol. 49, supplement 5, pp. 256S-263S, 2009.

[42] M. Maegele, R. Lefering, N. Yucel et al., "Early coagulopathy in multiple injury: an analysis from the german trauma registry on 8724 patients," Injury, vol. 38, no. 3, pp. 298-304, 2007. 


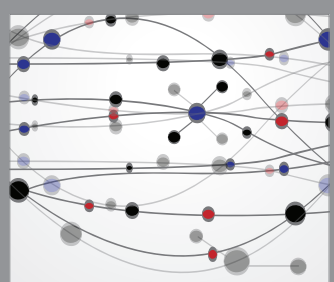

The Scientific World Journal
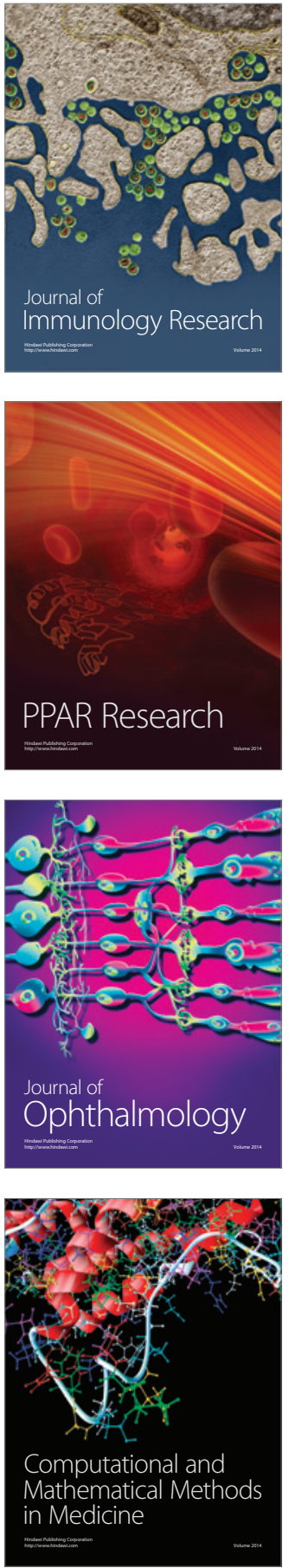

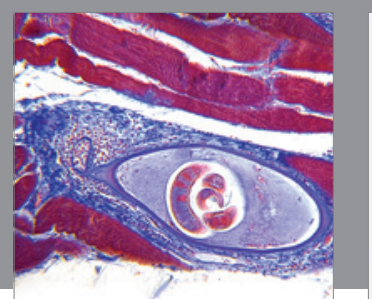

Gastroenterology Research and Practice

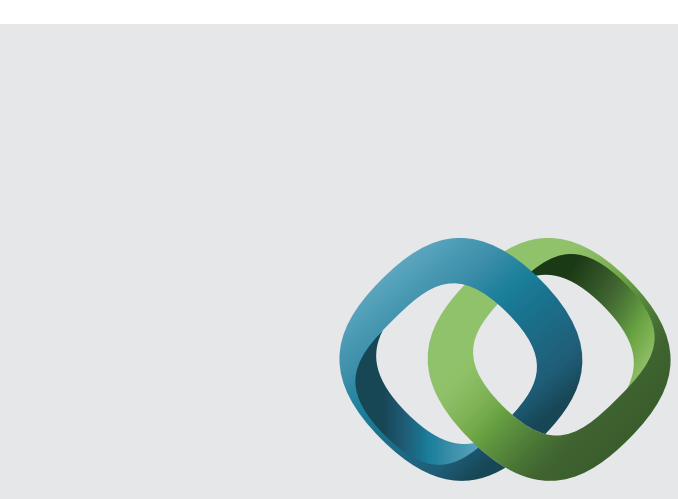

\section{Hindawi}

Submit your manuscripts at

http://www.hindawi.com
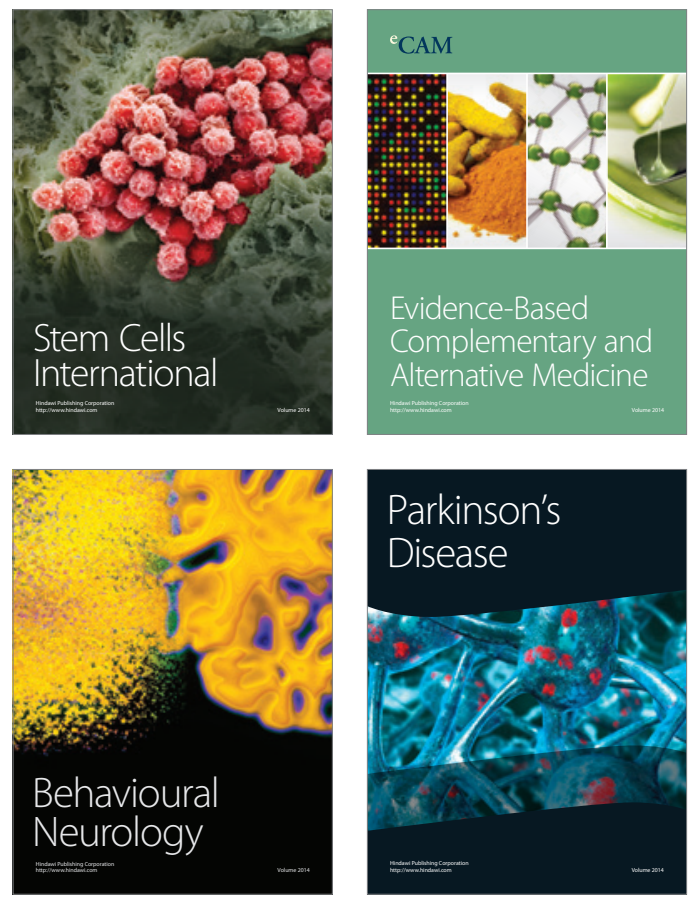
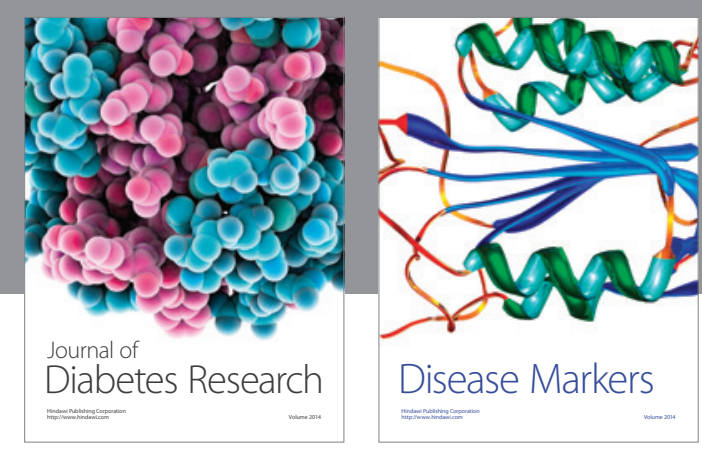

Disease Markers
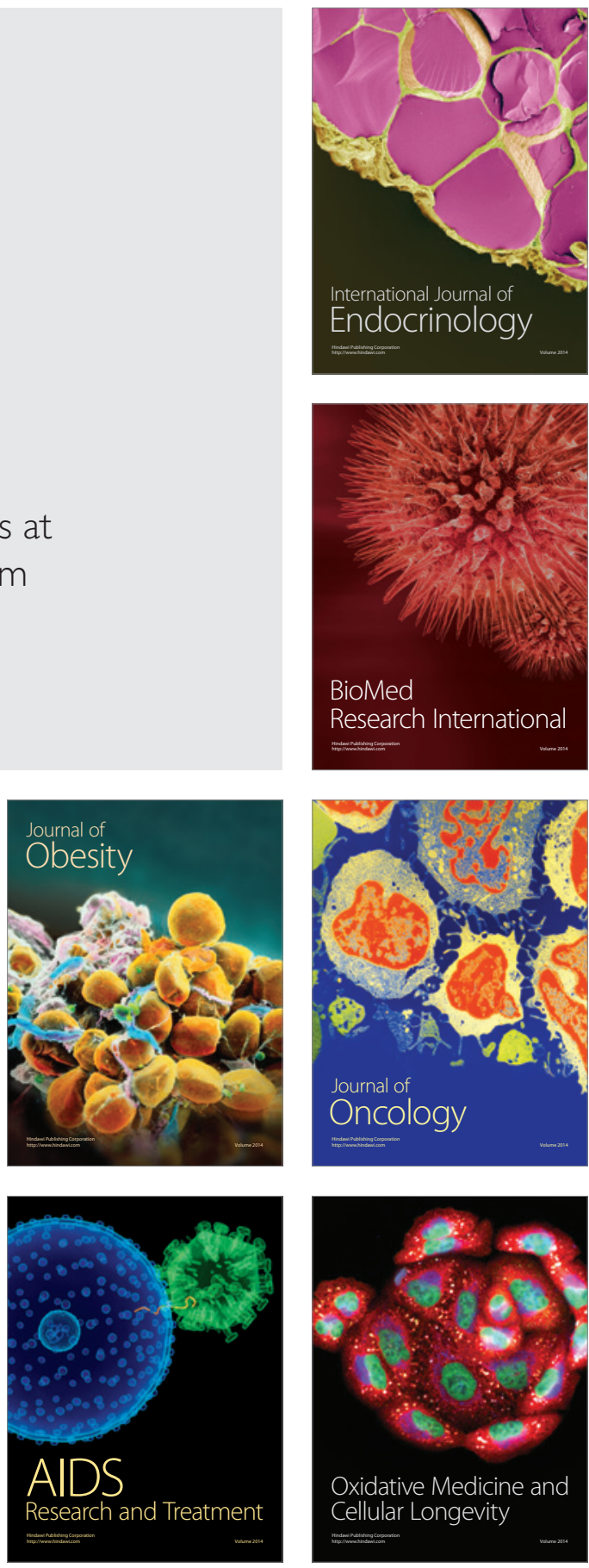